\title{
Acesso ao medicamento por via judicial na perspectiva dos usuários do Sistema Único de Saúde
}

Access to medicine through the courts from the perspective of users of the Brazilian Public Health System

\section{Virginia Oliveira Chagas}

Farmacêutica, Mestre em Ciências Ambientais e Saúde. Farmacêutica na Secretaria Municipal de Saúde de Jataí, Goiás, Brasil.

\section{Vanessa da Silva Carvalho Vila}

Enfermeira, Doutora em Enfermagem. Professora Adjunta da Pontifícia Universidade Católica de Goiás, Goiânia, Goiás, Brasil.

\section{Mércia Pandolfo Provin}

Farmacêutica, Doutora em Ciências da Saúde. Professora Adjunta da Universidade Federal de Goiás, Goiânia, Goiás, Brasil.

Resumo: $\mathrm{O}$ acesso aos medicamentos por meio de ações judiciais tem ocorrido com frequência nas três esferas de gestão do Sistema Único de Saúde, sendo legitimada pela garantia do direito universal e integral à saúde e é considerada uma via alternativa. Essa crescente demanda tem provocado muitas discussões entre juristas, gestores e profissionais de saúde, visto que pode interferir na gestão da assistência farmacêutica gerando gastos não previstos e comprometendo o orçamento para a saúde. Optou-se por desenvolver um estudo de caso qualitativo com o objetivo de descrever a perspectiva dos usuários sobre o acesso ao medicamento por via judicial no município de Jataí, Goiás. Participaram do estudo oito pacientes e seis familiares. Os dados foram coletados de janeiro a agosto de 2012, por meio de entrevista semiestruturada e análise documental dos processos judiciais. A experiência de busca pela via judicial esteve relacionada à falta de informação oferecida aos usuários, ao bom acolhimento dos profissionais do Judiciário e a resolutividade. Ficou evidente que, para o usuário, o acolhimento e a informação esclarecedora é imprescindível para tomada de consciência sobre a seriedade nesse processo de garantia do direito ao acesso ao medicamento. Em contrapartida, é preciso melhorar o processo comunicativo entre usuários e profissionais de saúde por meio de uma relação dialógica empática, respeitosa que possibilite a troca de experiências e o exercício da alteridade garantindo com isso ações integradas que atendam às necessidades individuais e coletivas para promoção da saúde.

Palavras-chave: Direito à saúde, assistência farmacêutica, acesso aos serviços de saúde.

Keywords: Right to health, pharmaceutical services, health services accessibility. 


\section{Introdução}

No Brasil, a partir da Constituição Federal de 1988, a saúde passou a ser reconhecida como direito social a ser garantida pelo Estado, por meio de políticas sociais e econômicas que têm como objetivo a promoção do bem-estar social, da plena cidadania e da qualidade de vida da população (Brasil, 1988).

O direito à saúde está relacionado com a elaboração de políticas públicas, que devem atender os principais problemas de saúde da população, visando não só a recuperação da saúde, como também sua prevenção e promoção. Neste contexto, a saúde deve ser garantida a todos de forma equânime visando ao atendimento integral (Brasil, 2011). O direito à assistência farmacêutica é considerado parte integrante do direito à saúde, cabendo, então, ao poder público formular e implementar políticas e ações que assegurem assistência terapêutica e farmacêutica integralmente aos cidadãos (Brasil, 1990a; Brasil, 1990b).

Entre os problemas vivenciados pelo cidadão brasileiro no sentido de garantir o direito à saúde, destaca-se a dificuldade de acesso aos medicamentos por parte de alguns em função de questões sociais e econômicas que impedem a segurança e a resolutividade do plano terapêutico. O direito à assistência farmacêutica depende de uma política pública e de recursos públicos para ser garantido.

Frente à dificuldade em ter acesso aos medicamentos prescritos para o efetivo tratamento das enfermidades, atualmente, o fenômeno da judicialização do acesso à saúde tem sido amplamente discutida no Brasil e entre as principais áreas que geram processos judiciais está a assistência farmacêutica (Sant'Ana et al., 2011).

O aumento das demandas judiciais, solicitando medicamentos, vem causando impactos nas políticas públicas e no orçamento destinado ao setor saúde, tendo em vista que os recursos públicos destinados à saúde são finitos e todos os cidadãos brasileiros possuem o mesmo direito à assistência farmacêutica. Desse modo, é importante que as necessidades individuais estejam inseridas dentro das políticas públicas (Marques, 2011).

As evidências científicas apontam que as lacunas assistenciais para o acesso ao medicamento e o fenômeno crescente do empoderamento individual e coletivo, têm promovido um movimento nacional de usuários que busca garantir o seu direito à saúde. Este fenômeno pode ser comprovado por meio do aumento do número de 
processos judiciais direcionados às Secretarias Municipais e Estaduais de Saúde em todo o país (Leite; Mafra, 2010; Pereira et al., 2010; Vieira; Zucchi, 2007).

Torna-se, assim, importante abordar essa questão, visto que um dos desafios do Sistema Único de Saúde (SUS) é garantir o acesso ao medicamento a todos os usuários. É importante e necessário discutir a atuação do Judiciário, na garantia do direito individual de alguns usuários que solicitam medicamentos, por meio de decisões judiciais, e seu impacto na política pública destinada a atender o coletivo, dentro do orçamento previsto para a saúde.

Considera-se importante fortalecer os estudos sobre a assistência farmacêutica no SUS na perspectiva dos usuários, com vistas a explicitar as percepções, as vivências e as suas experiências. Neste contexto, o presente estudo teve por objetivo analisar a perspectiva de usuários do SUS que recorreram à via judicial para ter acesso ao medicamento.

\section{Métodos}

Optou-se por desenvolver um estudo de caso interpretativo com usuários que recorreram à via judicial para ter acesso ao medicamento. Foram analisados os processos judiciais, movidos contra a Secretaria Municipal de Saúde de Jataí, Goiás, no período de janeiro a dezembro de 2010.

Foram realizadas entrevistas semiestruturadas no domicílio dos indivíduos que demandaram medicamentos por via judicial. Para a seleção dos sujeitos foram considerados os seguintes critérios: indivíduos com idade igual ou superior a dezoito anos, que foram responsáveis pela solicitação de pelo menos um medicamento por via judicial no município de Jataí. Por compreender que a vivência desse processo envolveu também o núcleo familiar, foram incluídos os membros da família que participaram do processo de solicitação do medicamento.

As entrevistas com os usuários e familiares foram conduzidas por meio de um roteiro que contemplou variáveis socioeconômicas (idade, nível de escolaridade, renda individual e familiar e ocupação). As questões norteadoras das entrevistas foram: Como foi para você ter acesso ao medicamento que você precisava? Qual o caminho você teve que percorrer para conseguir ter acesso ao medicamento? Por que foi necessário solicitar o medicamento por via judicial? Como foi a experiência de recorrer à via judicial para ter acesso ao medicamento? 
À medida que se aprofundou na leitura dos dados, procedeu-se à construção das unidades de significado. As unidades de significados são formadas por fragmentos textuais representativos de significados para os participantes, da realidade social e da abstração do pesquisador (Miles; Huberman, 1994; Straus; Corbin, 1998). As unidades semelhantes foram agrupadas em núcleos temáticos a partir da semelhança entre os significados.

O estudo foi aprovado pelo Comitê de Ética em Pesquisa da Pontifícia Universidade Católica de Goiás sob o protocolo № 1933/2011. Os participantes tiveram sua identidade protegida por nomes fictícios e assinaram termo de consentimento livre e esclarecido.

\section{Resultados e discussão}

Foram analisados os processos demandados por oito usuários, sendo cinco do sexo feminino e três do sexo masculino. A idade variou de 21 a 74 anos e o nível de escolaridade foi de 0 a 14 anos. A renda mensal individual de seis participantes foi de $R \$ 622,00$, um de $R \$ 2.367,00$ e outra não tinha renda. Entre os participantes, sete contribuíam com a renda mensal familiar.

Entre os familiares que participaram, quatro eram do sexo feminino e dois do sexo masculino. A idade variou de 24 a 68 anos. O nível de escolaridade foi de 12 anos, e a renda mensal individual variou de $R \$ 622,00$ a $R \$ 1.378,00$. Dos familiares, três eram funcionários públicos, duas do lar e uma era vendedora. Quanto ao grau de parentesco identificou-se uma filha, duas irmãs, dois esposos e uma mãe.

A análise interpretativa da experiência vivenciada por pacientes e por familiares para o acesso ao medicamento por via judicial, identificou uma trajetória marcada por um processo longo que envolveu a persistência, o apoio familiar e a necessidade de garantir o direito à saúde de pessoas que vivenciam condições crônicas que trazem limitações importantes para sua qualidade de vida. Foram observados dois possíveis itinerários no processo de acesso ao medicamento.

No primeiro itinerário, seis participantes mencionaram que inicialmente procuraram a Secretaria Municipal de Saúde de Jataí, para obter o medicamento. Após a constatação de que o(s) medicamento(s) prescrito(s) não estava(m) presente(s) na Relação Nacional de Medicamentos Essenciais (Rename) foi necessária a abertura do processo administrativo. Essa trajetória foi marcada, 
principalmente, pela demora e pela falta de acolhimento que motivaram o usuário a recorrer ao Ministério Público para propor uma ação judicial contra o município e, com prazo estabelecido, obter o medicamento mais rapidamente, de forma a não comprometer ainda mais o plano terapêutico e garantir a resolutividade do acesso.

No segundo itinerário, dois usuários procuraram diretamente o Ministério Público para ter acesso aos medicamentos prescritos. A partir disso, o Ministério Público entrou com a ação judicial contra o município e determinou um prazo para seu cumprimento. A Secretaria Municipal de Saúde adquiriu o medicamento por meio de compra direta ou por processo licitatório para que fosse dispensado ao usuário. Destaca-se que, nestes processos havia medicamentos que não estavam presentes na Rename, mas também, medicamentos previstos e que poderiam ser disponibilizados nas unidades de saúde com dispensação de medicamentos no município.

Neste contexto, foram identificados os seguintes núcleos temáticos: a experiência da tentativa de acesso ao medicamento via processo administrativo e a experiência de buscar o medicamento via processo judicial.

\section{A experiência da tentativa de acesso ao medicamento via processo administrativo}

Neste núcleo temático foram agrupadas as unidades de significado relacionadas à falta de informação fornecida aos usuários na busca pelo medicamento na via administrativa, o difícil acolhimento pelos profissionais e a demora sem resposta do processo administrativo.

Os participantes se queixaram da falta de informação e consideraram desrespeitoso o vaivém de informações sobre aonde ir e que documentos levar:

[...] você fica como se fosse uma bolinha de pingue-pongue! Muitas vezes, eles te mandam para a secretaria, a secretaria manda para a assistente social [...] (Gustavo).

Os participantes mencionaram a burocracia para montar o processo administrativo e reclamaram do tempo de espera para obter uma resposta:

[...] eu acho que é muita burocracia! Muita papelada! A gente que tem uma pessoa doente em casa não consegue esperar muito [...] demorou muito minha irmã ganhar o remédio! Com a assistente social foi mais ou menos nove meses [...] e não dava mais para esperar [...] (Irmã de Eliane). 
Destaca-se a importância dos profissionais envolvidos na via administrativa a fornecerem informações aos usuários que necessitam utilizar essa via de acesso ao medicamento. As informações devem ser claras e objetivas, principalmente sobre a documentação necessária, procurando desburocratizar o acesso.

O aumento da demanda por medicamentos, não inseridos em listas oficiais de financiamento público, a burocracia para ter acesso pela via administrativa, e a falta de profissionais preparados responsáveis pelo acolhimento dos usuários refletem a falta de planejamento das ações pelos gestores com relação a essa forma de acesso, e acaba interferindo na organização e resolutividade do serviço (Ronzani; Silva, 2008).

No estudo de Souza et al. (2008), a burocracia e a falta de acolhimento foram relatados pelos usuários em relação ao acesso na atenção básica. Os usuários se referiam ao atendimento desorganizado, com filas de espera, e o excesso de burocracia. Para esses usuários, o acolhimento está relacionado a atitudes pessoais e estruturais disponibilizadas de forma direta desde seu primeiro contato com o serviço.

\section{A experiência de buscar o medicamento via processo judicial}

A partir da compreensão da experiência vivenciada pelos participantes, relacionados à utilização da via judicial, verificou-se que no momento em que procuraram o Ministério Público, houve falha na informação. Esta falha também resultou em uma peregrinação do usuário até o profissional prescritor, para que fizesse um relatório médico mais detalhado e fundamentado, justificando o critério estabelecido para a utilização do medicamento. Apesar de existir um protocolo no Ministério Público para requerer uma ação judicial contra o município de Jataí, ainda existem falhas na informação ao usuário, que enxergaram isso como uma dificuldade:

[...] foi difícil pegar o relatório, porque o médico fez errado, fez a receita errado! Tinha que fazer de novo, sabe? Aí tinha que estar voltando lá, correndo atrás porque tem que ser certinho, né? Tem que fazer o relatório e falar o que é a doença, o que precisa, como que é, como é que não é, esse tipo de coisa assim! (Irmã de Lázaro).

A informação é o elemento central para a compreensão das dimensões do acesso. Estar bem informado é resultado do processo de comunicação entre o sistema de saúde e o indivíduo, além de determinar a lacuna entre a oportunidade de utilizar e a real utilização dos serviços de saúde (Sanchez; Ciconelli, 2012). 
O acolhimento por parte dos profissionais do Ministério Público envolvidos no acesso ao medicamento por via judicial foi mencionado por unanimidade por todos participantes do estudo.

[...] os outros humilham (via administrativa), dão má resposta, falam as coisas pra gente, é muito difícil, mas na promotoria eu fui muito bem recebida![...] (Luiza).

Os usuários narraram ser bem atendidos e não se sentiram humilhados como na via administrativa. O vínculo do acolhimento com os profissionais está relacionado com a tomada de decisão quando não se é possível atender à necessidade do usuário e não se tem disponibilidade para dialogar com ele, utilizando uma linguagem acessível. Esse vínculo amplia as possibilidades da participação do usuário na tomada de decisão (Pinafo; Lima; Baduy, 2008).

Observou-se que os usuários correlacionaram a via judicial como algo resolutivo, principalmente quando se referiam ao menor tempo de espera quando comparado ao tempo da via administrativa.

[...] o ministério público vê a necessidade de como o aposentado sobrevive com uma carga enorme de medicamentos! [...] talvez $80 \%$ do salário do idoso é para comprar medicamentos! Então o que sobra? Agora se for tirando tudo só do salário, ele morre de fome! Então nesse caso aí, o ministério público foi o caminho mais ágil! Se todo mundo já fosse direto ao ministério público seria resolvido essa dificuldade de ter que percorrer um, dois, três caminhos! (Gustavo).

O estudo de Sant'ana et al. (2011) também descreveu a via judicial como sendo mais rápida e menos burocrática, sendo, portanto, mais atrativa aos usuários. Isso contribuiu para uma satisfação deles. No estudo de Trad e Esperidião (2009) os usuários adotaram postura de gratidão pelo serviço recebido diante da ampliação do acesso aos serviços e aos medicamentos, por desconhecerem o direito constitucional à saúde. Poucos usuários reconhecem os progressos no sistema de saúde como um direito de cidadania.

\section{Considerações finais}

Este estudo permitiu descrever a realidade vivenciada por indivíduos que recorreram à via judicial para ter acesso aos medicamentos no município de Jataí, Goiás.

Os resultados sugerem a necessidade de humanização do atendimento, melhorando o acolhimento aos usuários pelos profissionais envolvidos no acesso ao 
medicamento pela via administrativa, que tem se tornado uma via alternativa para as demandas judiciais e tem provocado muitas discussões entre juristas, gestores e profissionais de saúde, visto que este tipo de demanda pode interferir na gestão da assistência farmacêutica gerando gastos não previstos e comprometendo o orçamento para a saúde.

Por outro lado, observou-se que pela via judicial, os usuários foram acolhidos pelos profissionais envolvidos, e foi possível compreender que este processo gerou satisfação e gratidão por parte desses usuários, que identificaram esta via como algo resolutivo, diferente da via administrativa.

É preciso considerar a necessidade de aprimoramento dos protocolos de acesso aos medicamentos pela via administrativa com o preparo para humanização do atendimento, e dos protocolos existentes no Ministério Público para a proposição de ação judicial, como também o preparo dos profissionais envolvidos a fornecerem informações claras aos usuários.

Ficou evidente que para o usuário, o acolhimento e a informação esclarecedora são imprescindíveis para a tomada de consciência sobre a seriedade do processo administrativo a fim de se garantir o direito ao acesso à saúde. Em contrapartida, é preciso melhorar o processo comunicativo entre usuários e profissionais de saúde por meio de uma relação dialógica empática, respeitosa que possibilite a troca de experiências e o exercício da alteridade, garantindo, com isso ações integradas que atendam às necessidades individuais e coletivas para a promoção da saúde.

Os gestores públicos deverão favorecer a atuação das equipes de saúde, por meio da elaboração e a implementação de protocolos para dispensação de medicamentos, que considerem principalmente as diretrizes para o acolhimento dos usuários em suas necessidades de cuidados à saúde.

\section{Referências}

BRASIL. Conselho Nacional de Secretários de Saúde. Sistema Único de Saúde. Brasília: CONASS, 2011. 291 p.

BRASIL. Constituição da República Federativa do Brasil de 1988. Diário Oficial da União, Brasília, DF, 5 out. 1988.

BRASIL. Lei no 8.080, de 19 de setembro de 1990. Dispõe sobre as condições para a promoção, proteção e recuperação da saúde, a organização e o funcionamento dos 
serviços correspondentes e dá outras providências. Diário Oficial da União, Brasília, DF, 20 set. 1990a.

BRASIL. Lei no 8.142, de 28 de dezembro de 1990. Dispõe sobre a participação da comunidade na gestão do Sistema Único de Saúde (SUS) e sobre as transferências intergovernamentais de recursos financeiros na área da saúde e dá outras providências. Diário Oficial da União, Brasília, DF, 31 dez. 1990b.

LEITE, Silvana Nair e MAFRA, Ana Cristina. C. Que direito? Trajetórias e percepções dos usuários no processo de acesso a medicamentos por mandados judiciais em Santa Catarina. Ciência \& Saúde Coletiva, Rio de Janeiro, 15(suppl.1):1665-1672, 2010.

MARQUES, Silvia Badim. O direito ao acesso universal a medicamentos no Brasil: diálogos, a política e a técnica médica. Tese (doutorado). São Paulo, Universidade de São Paulo, Faculdade de Saúde Pública, 2011.

MILES, Matthew B. e HUBERMAN, A. Michael. Qualitative data analysis: an expanded sourcebook. 2. ed. London: Sage Publications, 1994.

PEREIRA, Januária Ramos [et al]. Análise das demandas judiciais para o fornecimento de medicamentos pela Secretaria de Estado da Saúde de Santa Catarina nos anos de 2003 e 2004. Ciência \& Saúde Coletiva, Rio de Janeiro, 15(suppl. 3):3551-3560, 2010.

PINAFO, Elisângela e LIMA, J Josiane Vivian de Camargo e BADUY, Rossana Staevie. Acolhimento: concepção dos auxiliares de enfermagem e Percepção de usuários em uma unidade de saúde da família. Espaço para a Saúde, Londrina, 9(2):17-25, 2008.

RONZANI, Telmo Mota e SILVA, Cristiane de Mesquita. O Programa Saúde da Família segundo profissionais de saúde, gestores e usuários. Ciência \& Saúde Coletiva, Rio de Janeiro, 13(1):23-34, 2008.

SANCHEZ, Raquel Maia e CICONELLI, Rosana Mesquita. Conceitos de acesso à saúde. Revista Panamericana de Salud Pública, 31(3): 260-268, 2012.

SANT'ANA, João Mauricio Brambati [et al]. Racionalidade terapêutica: elementos médico-sanitários nas demandas judiciais de medicamentos. Revista de Saúde Pública, São Paulo, 45(4):714-721, 2011.

SOUZA, Elizabethe Cristina Fagundes de [et al]. Acesso e acolhimento na atenção básica: uma análise da percepção dos usuários e profissionais de saúde. Cadernos de Saúde Pública, Rio de Janeiro, 24(suppl. 1):S100-S110, 2008

STRAUSS, Anselm e CORBIN, Juliet. Pesquisa qualitativa: técnicas e procedimentos para o desenvolvimento de teoria fundamentada. 2. ed. Porto Alegre: Artmed, 1998.

TRAD, Leny Alves Bomfim e ESPERIDIÃO, Monique Azevedo. Gestão participativa e corresponsabilidade em saúde: limites e possibilidades no âmbito da Estratégia de Saúde da Família. Interface, Botucatu, 13(suppl.1):557-570, 2009.

VIEIRA, Fabiola Sulpino e ZUCCHI, Paola Distorções causadas pelas ações judiciais à política de medicamentos no Brasil. Revista de Saúde Pública, São Paulo, 41(2):214-222, 2007. 